Demographic data, clinical manifestations, laboratory evaluation, antibiotic therapy, duration of hospitalization and clinical evolution were analyzed.

Results During the total of this period, there were 4163 hospitalizations in the Department of Pediatrics, of which 34 $(0.8 \%)$ for cases of impetigo. Of these cases, 19 (56\%) corresponded to bullous impetigo and 15 (44\%) to non-bullous. The proportion of impetigo cases at admission ranged from $0 \%$ in 2012 to 2013, up to $1.5 \%$ of hospital admissions in 2016. Most (94\%) were term newborns, 50\% male, with a mean age at diagnosis of 12.7 days. Neonatal onphalitis was the most commonly associated pathology in 41\%. In only $26 \%$ of cases, culture of the pus or bullous fluidwas performed. The most frequent agent was Staphylococcus aureus, identified in $63 \%$ of positive cultures. The most used antibiotic therapy $(50 \%)$ was the association of flucloxacillin with gentamicin. The evolution was favorable, with complete resolution of the clinical manifestations during hospitalization, in all cases.

Conclusions Our study shows a demographic, clinical and laboratory characterization coincident with that described in the current literature. The increase in the proportion of hospitalizations due to impetigo over the years raises the possibility that the incidence of the pathology is increasing or that there is an increase in the severity of the cases. These data should be an alert on the possible influence that the adequacy of the care provided to the newborn, bothinside or outside the hospital, may have in terms of public health.

\section{P474 EXTRA-HEPATIC BILE DUCT HAMARTOMA - AN UNUSUAL CAUSE FOR ANTERIOR ABDOMINAL WALL DEFECT}

${ }^{1}$ Abhidhamma Kaninde*, ${ }^{2}$ Sheiniz Giva, 3,4 Maragaret M Moran, ${ }^{5,6}$ Salvatore Cascio, ${ }^{7}$ Erinn S McGrath, J Maureen ${ }^{8,9,10} \mathrm{O}^{\prime}$ Sullivan. ${ }^{1}$ Dept.of Neonatology, The Rotunda Hospital, Dublin, Ireland; ' ${ }^{2}$ Dept. of Neonatology, The Rotunda Hospital , Dublin, Ireland; ${ }^{3}$ Dept. of Neonatology, The Rotunda Hospital, Dublin, Ireland; ${ }^{4}$ Dept. of Neonatology, Children's University Hospital ,Templestreet, Dublin, Ireland; ${ }^{5}$ Dept. of Paediatric Surgery, Children's University Hospital, Templestreet, Dublin , Ireland; ' ${ }^{6}$ Dept. of Paediatric Surgery, Our Lady's Children's Hospital, Crumlin, Dublin, Ireland; 'Dept.of Pathology, Our Lady's Children's Hospital, Crumlin, Dublin, Ireland; ${ }^{8}$ Dept. of Pathology, Our Lady's Children's Hospital, Crumlin, Dublin , Ireland; ' University of Dublin, Trinity College , , Dublin, Ireland; ${ }^{10}$ Principal Investigator, The National Children's Research Centre,Our Lady's Children's Hospital, Crumlin, Dublin, Ireland

\subsection{6/archdischild-2019-epa.810}

We report a case of a baby girl born at 35 weeks gestation with an unusual para-umbilical lesion associated with an anterior abdominal wall defect. Shortly after birth, she was noted to have a small polypoid lesion to the right of her umbilicus. The lesion measured around $1 \mathrm{~cm}$ in length and had a small area of ulceration at the tip. Her systemic examination was otherwise normal.

Abdominal ultrasound examination was suggestive of a defect in the anterior abdominal wall. An artery and vein could be seen passing through the defect into the lesion. A rounded echogenic structure was noted to be passing through the abdominal wall defect consistent with a loop of bowel. At laparotomy, a hamartomatous lesion was seen with an artery and vein extending back to the liver. The bowel was intact and there was no bowel within the lesion. The lesion was excised, with ligation of the blood vessels, and closure of the abdominal wall defect. Screening for other midline anomalies revealed a left-sided grade 1 intraventricular haemorrhage and a small fenestrated atrial septal defect. Genetic analysis showed a normal microarray and no evidence of Beckwith-Wiedemann syndrome.

Histopathological examination of the tissue showed extensively ulcerated skin. The outline was polypoid with granulation tissue as a base. Deep to this were bile ducts, venules, and arterioles. These features were hamartomatous suggesting an extra-hepatic bile duct hamartoma. The lesion was malformative and not neoplastic.

Discussion While it is unusual to find biliary tree hamartomas presenting in the neonatal period, as most of them are asymptomatic and insidious, the extra-hepatic location of this hamartoma is extremely rare. Redston et al. reported on autopsy findings of 2843 patients and calculated the prevalence of biliary hamartomas as $5.6 \%$ in adults and $0.9 \%$ in children. They also found a high incidence of biliary hamartomas with autosomal dominant polycystic kidney disease. They can be solitary or multiple. Multiple bile duct hamartomas are also known as Von Meyenburg complexes. They are a benign ductal plate malformation of smaller interlobular ducts. Although these lesions are often described as benign anecdotally there are reports mentioning malignant transformation.

Conclusion We report a very rare case of extra-hepatic bile duct hamartoma presenting as an anterior abdominal wall defect in the neonatal period.

\section{P475 REVIEW OF THE MANAGEMENT OF BILIOUS VOMITING IN TERM NEONATES AND IDENTIFY DIFFERENCES IN MANAGEMENT OUTSIDE WORKING HOURS IN CUMH}

${ }^{1}$ Carol Stephens*, ${ }^{2}$ liam o'connell. ${ }^{1}$ Neonatology Department, cork, Ireland; ${ }^{2}$ Neonatology Department, Cork, Ireland

\subsection{6/archdischild-2019-epa.811}

Bilious vomiting is a common reason for admission to the neonatal unit. In the majority, the episode is benign. In a small minority, there is serious underlying pathology which is time critical. Identification and differentiation between these entities is crucial but challenging. Management is variable amongst centres and often case specific and certainly always time critical. When a pathological cause is suspected, occasionally it is warranted to transfer the sick neonate to a surgical unit without the delay of local investigations.

Methods This was a retrospective review in CUMH over seven years.

Inclusion criteria were all infants admitted directly to the unit with bilious vomiting.

Exclusion criteria included infants who were admitted for a different primary concern including premature infants.

Admission Logbook of unit identified infants, online reporting systems were used to review discharge summaries. Online laboratory and radiology database were accessed.

Results 213 infants were included. Management varied throughout the years. Earlier, most infants had a PFA, Septic Work Up (SWU) and contrast study in contrast to the latter years where most had a PFA and a SWU. We postulate in the latter years there was a lower threshold for admission on the background of a neonatal death and subsequently more infants were conservatively managed and observed in the unit.

$22 \%$ had contrast studies out of hours, $18 \%$ were subsequently transferred to a surgical unit in light of a positive 
finding. Positive findings included microcolon, volvulus, Hirschprungs and obstruction. The majority of infants were discharged with no complications.

Discussion Bilious vomiting is synonymous with intestinal obstruction and should be considered this until proven otherwise . Management is time critical given the potential consequences of a volvulus and the ischaemic threat to the bowel with the window of opportunity being approximately six hours (3) A national guideline is now warranted.

\section{P476 SIRENOMELIA (MERMAID SYNDROME) - A RARE CONGENITAL DISORDER}

${ }^{1}$ Muhammad Imran Riazat*, 'Jamaleddin Abujennah, ${ }^{2}$ Bharti Kewlani, ${ }^{1}$ Farhana Sharif. ${ }^{1}$ Mullingar Regional Hospital, Mullingar, Ireland; ${ }^{2} R C S I$, Dublin, Ireland

10.1136/archdischild-2019-epa.812

Background Sirenomelia or mermaid syndrome is an extremely rare congenital disorder involving the lower spine and lower limbs. Although usually fatal in the newborn period, survival in a handful of cases beyond infancy have been reported. We would like to present a new born with sirenomelia and multiple anomalies brought in to our hospital soon after birth.

Case report A 40 year old Romanian lady G 17, P 16, Ab 0, known diabetic poorly controlled on insulin, delivered at 38 weeks gestation in an ambulance en route to the hospital. On arrival, she was taken directly to the labor ward with ongoing resuscitation efforts with bag and mask and CPR carried out by the paramedics. The baby was noted to be cyanosed and in respiratory distress. Severe congenital abnormalities were obvious and included flattened dysmorphic features, low set malformed ears, upward slanting palpebral fissures, flattened nose, receding chin, short neck, small thoracic cage, ambiguous genitalia, absent anal opening and fused lower limbs. The feet however, were separate.

The mother was originally booked in a tertiary referral center where she had undergone antenatal scanning and was counselled on the poor prognosis due to multiple abnormalities detected including but not limited to anhydramnios (that could result in pulmonary hypoplasia), absent left kidney, right cystic dysplastic kidney and cardiac malformation. When the mother was brought in by ambulance into our hospital, we were not aware of the management plan. The baby was therefore intubated and started on positive pressure ventilation as per the protocol. Shortly after, we realized futility after resuscitation became obvious. We received more information regarding the poor prognosis and consequently, extubated the baby at 18 minutes of life and handed over the baby to the mother for comfort care.

Genetic blood tests for microarray and karotyping were carried out and revealed female karyotype with normal microarray. Her skeletal survey was requested which showed caudal regression and absence of vertebras after S1, malformed pelvis and soft tissue fusion of both lower limbs, however feet were separated.

Conclusion Sirenomelia is an extremely rare and usually fatal congenital malformation. Maternal diabetes, smoking and heavy metal exposure have been implicated as possible etiologies. Therefore, in our opinion poor glycemic control prior to conception and during gestation (especially during the first trimester) could have contributed to this condition.

\section{P477} TWO CASES OF LATE ONSET GBS INFECTION DESPITE POST NATAL BENZYLPENICILLIN ADMINISTRATION

${ }^{1}$ Simon Piggott*, 'Edina Moylett, 'Ethel Ryan, ${ }^{2}$ Una Ni Riain. ${ }^{1}$ Academic Department of Paediatrics, National University of Ireland, Galway, Galway, Ireland; 'Department of Microbiology, University Hospital Galway, Galway, Galway, Ireland

\subsection{6/archdischild-2019-epa.813}

Aim Streptococcus agalactiae (Group B streptococcus, GBS) is the leading cause of invasive infection among neonates and young infants in the developed world. The prevention of late onset GBS disease (LOD) remains elusive. We present two cases of LOD, one near fatal with significant morbidity, despite early treatment with benzylpenicillin in each case.

Method Following confirmation of GBS LOD, the medical record for each infant was reviewed at University Hospital Galway. Relevant clinical and laboratory information was recorded.

Results Case 1, following an intrapartum high vaginal swab positive for GBS with a clinical suspicion for chorioamnionitis at time of delivery, an otherwise well infant (born at $28+6$ weeks) was treated empirically with a 5 day course of IV benzylpenicillin and gentamicin despite sterile blood cultures. The infant's neonatal intensive care unit (NICU) course was uneventful until day of life (DOL) 25 when following a clinical deterioration necessitating a sepsis evaluation, the infant's blood culture isolated GBS, the infant made a full recovery. Case 2, a singleton female infant (born at $31+6$ weeks) was treated empirically with a 48 hour course of IV benzylpenicillin and gentamicin owing to an intra-uterine death of unknown cause at 18 weeks during a triplet pregnancy. There were no positive swabs/clinical suspicion for intra-partum infection with neonatal sterile blood cultures following delivery. Standard NICU care was carried out until DOL 9 when the infant developed overwhelming sepsis with GBS positive blood cultures and was transferred to a tertiary NICU centre for further care. The infant survived with significant morbidities. In both of our cases, multi-locus sequence typing and serotyping allowed accurate identification of isolates. Our chosen cases demonstrate the continued prevalence of GBS and the recognised limitations of prophylactic antibiotic use with both of the infants developing GBS infection between days 7-89 of life despite initial antibiotic administration.

Conclusion Antenatal risk-based guidelines and antibiotic prophylaxis are effective in ameliorating early onset GBS infection but are inadequate for combating LOD. Admittedly, the pathogenesis of LOD remains elusive and the route of acquisition is still unclear, possibly vertically from mother or horizontally from environmental sources. Our cases support each of these theories. Postnatal antibiotic therapy does not protect against LOD highlighting the potential role for vaccination in LOD prevention.

\section{P478 RARE CASE OF SEVERE THROMBOCYTOPENIA AT BIRTH ASSOCIATED WITH RHESUS DISEASE OF THE NEWBORN}

${ }^{1}$ Alina G Deliu*, Dr Melany Cotter ${ }^{2}$, Dr ${ }^{3}$ JB Letshwiti. 'Mayo University Hospital , Castlebar, Ireland; '2Our Lady's Children's Hospital, Crumlin , Dublin, Ireland; ${ }^{3}$ Galway University Hospital, Galway, Ireland

\subsection{6/archdischild-2019-epa.814}

Aims Rhesus isoimmunization (RI) is becoming relatively uncommon with the introduction of Rho (D) immune globulin 